\title{
Enhancement of oral bioavailability of cyclosporine A: comparison of various nanoscale drug-delivery systems
}

This article was published in the following Dove Press journal:

International Journal of Nanomedicine

28 October 2014

Number of times this article has been viewed

\author{
Kai Wang ${ }^{1-3}$ \\ Jianping $\mathrm{Q}^{1}$ \\ Tengfei Weng ${ }^{1,2}$ \\ Zhiqiang Tian' \\ Yi Lu' \\ Kaili $\mathrm{Hu}^{4}$ \\ Zongning Yin $^{2}$ \\ Wei Wu'
}

'School of Pharmacy, Fudan University, Key Laboratory of Smart Drug Delivery of Ministry of Education, Shanghai, People's Republic of China; ${ }^{2}$ West China School of Pharmacy, Sichuan University, Chengdu, Sichuan, People's Republic of China; ${ }^{3}$ Tropical Crops Genetic Resources Institute, Hainan Provincial Engineering Research Center for Blumea Balsamifera, Chinese Academy of Tropical Agricultural Sciences, Danzhou, Hainan, People's Republic of China; ${ }^{4}$ Murad Research Center for Modernized Chinese Medicine, Shanghai University of Traditional Chinese Medicine, Shanghai, People's Republic of China

Correspondence: Jianping Qi Department of Pharmaceutics, School of Pharmacy, Fudan University, 826 Zhangheng Road, Shanghai 20I203, People's Republic of China

Tel +86 2I 5I980084

Fax +86 21 51980084

Email qijianping@fudan.edu.cn

Zongning Yin

West China School of Pharmacy, Sichuan University, Chengdu 6I004I, Sichuan,

People's Republic of China

Email yzn@scu.edu.cn
Abstract: A variety of nanoscale delivery systems have been shown to enhance the oral absorption of poorly water-soluble and poorly permeable drugs. However, the performance of these systems has seldom been evaluated simultaneously. The aim of this study was to compare the bioavailability enhancement effect of lipid-based nanocarriers with poly(lactic-co-glycolic acid) (PLGA) nanoparticles (NPs) to highlight the importance of the lipid composition, with cyclosporine A (CyA) as a model drug. CyA-loaded PLGA NPs, nanostructured lipid carriers (NLCs), and self-microemulsifying drug-delivery systems (SMEDDS) were prepared. The particle size of PLGA NPs $(182.2 \pm 12.8 \mathrm{~nm})$ was larger than that of NLCs $(89.7 \pm 9.0 \mathrm{~nm})$ and SMEDDS $(26.9 \pm 1.9 \mathrm{~nm})$. All vehicles are charged negatively. The entrapment efficiency of PLGA NPs and NLCs was $87.6 \% \pm 1.6 \%$ and $80.3 \% \pm 0.6 \%$, respectively. In vitro release tests indicated that the cumulative release of CyA was lower than $4 \%$ from all vehicles, including Sandimmun Neoral ${ }^{\circledR}$, according to the dialysis method. Both NLCs and SMEDDS showed high relative oral bioavailability, $111.8 \%$ and $73.6 \%$, respectively, after oral gavage administration to beagle dogs, which was not statistically different from commercial Sandimmun Neoral ${ }^{\circledR}$. However, PLGA NPs failed to achieve efficient absorption, with relative bioavailability of about $22.7 \%$. It is concluded that lipid-based nanoscale drug-delivery systems are superior to polymeric NPs in enhancing oral bioavailability of poorly water-soluble and poorly permeable drugs.

Keywords: cyclosporine A, PLGA nanoparticle, nanostructured lipid carrier, self-microemulsifying drug-delivery systems, bioavailability

\section{Introduction}

It is well known that the oral route is the first choice clinically for drug administration due to better patient compliance. However, most new chemical entities (approximately $60 \%$ of drugs) coming directly from chemical synthesis are poorly water-soluble, ${ }^{1}$ and a large fraction of them are even poorly permeable across the biomembrane in the gastrointestinal tract (GIT). Drug substances with low solubility and poor permeability exhibit extremely low bioavailability after oral administration, which may result in limited therapeutic potential and thus insufficient curative effects. ${ }^{2}$ According to the definition of Biopharmaceutics Classification System (BCS), ${ }^{3}$ these drugs are categorized as BCS IV. Consequently, BCS IV drugs yield the most challenging absorption problems, ${ }^{4}$ such as low drug dissolution, efflux by transporters in the gut wall, and first-pass effect by metabolic enzymes. ${ }^{5}$

Fortunately, smart drug carrier systems are emerging to handle each difficult problem. Colloidal carriers, whether directly nanosized or incorporated into polymeric or lipidic nanoparticles (NPs) (eg, nanocrystals, ${ }^{6}$ poly(lactic-co-glycolic acid) (PLGA) $\mathrm{NPs}^{7}{ }^{7}$ and microemulsions $\left.\mathrm{s}^{8,9}\right)$, are proved to facilitate adhesive interaction within the 
mucosa and remain in the intestinal tract until the release of the loaded drug or their absorption in an intact particulate form. They can help to deliver drugs with poor aqueous solubility, low permeability, and extensive first-pass metabolism, because of the special mechanisms of uptake through the GIT. For example, NPs bind to the apical membrane of the $\mathrm{M}$ cells, followed by rapid internalization and shuttling to lymphocytes, wherein size and surface charge play a crucial role for their uptake. ${ }^{10,11}$ Apart from these common advantages, different colloidal carriers have distinguishing features of their own. Some polymeric NPs, such as PLGA NPs, improve oral absorption of drugs through increasing dispersion of drugs and epithelia endocytosis. ${ }^{12}$ Nevertheless, it is generally thought that digestion products of lipid-based nanocarriers, such as lipid NPs and nanoemulsion, can solubilize lipophilic drugs and that the presence of endogenous bile salts may alter the intrinsic permeability of the intestinal membrane, leading to increased absorption via paracellular or transcellular routes. ${ }^{13-15}$

Both lipid-based nanocarriers and polymeric NPs were suggested as distinguished delivery systems in improving oral absorption of BCS IV drugs, according to many reports. ${ }^{16,17}$ Some reports have suggested that lipid-based nanocarriers are superior to polymeric NPs in improving oral bioavailability of BCS IV drugs. ${ }^{18}$ These comparisons are based on data from different research groups, however, and are therefore affected by choice of animal models, experimental design, and other experimental conditions. Thus, in order to clarify the differences between lipid-based nanocarriers and polymeric NPs and to provide valuable information for the formulation design of BCS IV drugs, bioavailability comparisons within the same experimental condition is necessary.

This study utilized cyclosporine A (CyA), which is a cyclic polypeptide containing eleven amino acids and used as immunosuppressive, as a model drug. CyA is classified as a BCS IV drug due to its physicochemical properties, including high lipophilicity, polar surface area, and molecular weight. ${ }^{19-21}$ The factors impeding the absorption of CyA include the narrow absorption window in the upper gut, P-glycoprotein efflux from enterocytes, and extensive presystemic metabolism in the wall and liver. ${ }^{22,23}$ To date, how to improve CyA's in vivo performance has been a widespread concern, and various oral drug carriers have emerged, such as solid dispersion, ${ }^{19,24,25}$ nanosuspension, ${ }^{26}$ liposomes, ${ }^{27}$ lipid NPs, ${ }^{28}$ self-microemulsifying drug-delivery system (SMEDDS), ${ }^{16}$ and PLGA NPs. ${ }^{17}$ Although each of these were reported to significantly increase CyA oral bioavailability, only the SMEDDS of CyA (Sandimmun Neoral ${ }^{\circledR}$ ) was successfully marketed, revealing the superiority of lipidbased nanocarriers.

For this study selected CyA as the BCS IV drug model and beagle dogs as the animal model to compare the relative bioavailability of PLGA NPs (polymeric NPs) with two kinds of lipid-based nanocarriers (SMEDDS and nanostructured lipid carriers [NLCs]), with Sandimmun Neoral ${ }^{\circledR}$ used as a reference.

\section{Materials and methods Materials}

CyA was obtained from the Pharmaceutical Factory of Sichuan Institute of Antibiotic Industries (Chengdu, People's Republic of China). PLGA (Lakeshore Biomaterials ${ }^{\mathrm{TM}}$ 5050 DLG 2A, inherent viscosity: $0.20 \mathrm{dL} / \mathrm{g}$ ) was kindly gifted from Evonik Degussa Co., Ltd (Shanghai, People's Republic of China). Polyvinyl alcohol (average molecular weight $=13-23 \mathrm{kDa}$ ) was purchased from Sigma-Aldrich (St Louis, MO, USA). Precirol ATO 5 and Captex 100 were kindly provided by Gattefossé Co. (Saint Priest, Cedex, France) and Abitec Co. (OH, USA), respectively. Polysorbate 80 (Tween-80) was supplied by Shenyu Pharmaceutical and Chemical Co., Ltd (Shanghai, People's Republic of China). Oleoyl macrogolglycerides (Labrafil M ${ }^{\circledR} 1944$ CS) and diethylene glycol monoethyl ether (Transcutol $\mathrm{P}^{\circledR}$ ) were kindly gifted from Gattefossé Corporation (Brittany, France). Ethoxylated castor oil (Cremophor ${ }^{\circledR}$ EL) was obtained from BASF Corporation (Ludwigshafen, Germany). High-performance liquid chromatography (HPLC)-grade methanol and acetonitrile were purchased from Tedia (Carson City, CA, USA). Deionized water was prepared using a Milli-Q purification system (Millipore, Billerica, MA, USA). All other chemicals were of analytical grade and used as received.

\section{Preparation of PLGA nanoparticles}

PLGA NPs were prepared by a modified emulsion solventevaporation method. ${ }^{10,30}$ Briefly, $30 \mathrm{mg}$ of CyA and $150 \mathrm{mg}$ of PLGA were dissolved in $3 \mathrm{~mL}$ of ethyl acetate. The mixed solution was added dropwise to $3 \mathrm{~mL}$ of $1.5 \%$ polyvinyl alcohol solution under constant stirring to obtain the primary emulsion. The primary emulsion was then homogenized for 1 minute with the help of an ultrasonic machine (JY92-IIDN, NINGBO Scientz Biotechnology Co., Ltd, People's Republic of China). Afterwards, NPs were formed through adding 25 $\mathrm{mL}$ of water under 1,000 rpm stirring. The obtained suspension was kept overnight with moderate stirring to evaporate ethyl acetate. 


\section{Preparation of nanostructured lipid carriers}

NLC suspensions were prepared by the melting-emulsification technique, according to our previously described procedures with minor modifications. ${ }^{29}$ In brief, $1.0 \mathrm{~g}$ solid lipids (Precirol ATO 5) and $2.0 \mathrm{~g}$ liquid lipids (Captex100) were melted at $65^{\circ} \mathrm{C}$, in which $80 \mathrm{mg}$ CyA was dissolved. The CyA-containing melt was then dispersed in $50 \mathrm{~mL}$ water containing $1.4 \%(\mathrm{w} / \mathrm{v})$ Tween 80 under high-speed stirring using an Ultra-Turrax blender (QilinBeier, Jiangsu, People's Republic of China) at the same temperature. The obtained coarse emulsion was further homogenized by microfluidizer (Nano DeBEE, USA) applying four circles under 20,000 psi. The CyA-loaded NLCs were obtained when the homogenized emulsion was cooled to room temperature.

\section{Preparation of CyA-loaded self- microemulsifying drug-delivery system}

The formulation of CyA SMEDDS was prepared according to previously reported method by our group. ${ }^{30}$ In brief, CyA (160 mg) was dissolved in the mixture of $300 \mathrm{mg}$ Labrafil M 1944 CS, $466.7 \mathrm{mg}$ Cremophor EL, and $233.3 \mathrm{mg}$ Transcutol P. The final mixture was vortex-mixed, and then observed visually to identify the formation of SMEDDS.

\section{Measurement of particle size and zeta potential}

The Zetasizer Nano ${ }^{\circledR}$ (Malvern Instruments, Malvern, UK) equipped with a $4 \mathrm{~mW} \mathrm{He}-\mathrm{Ne}$ laser $(633 \mathrm{~nm})$ was employed to measure particle size and zeta potential at $25^{\circ} \mathrm{C}$. The NLCs and PLGA NPs were diluted 15-fold with deionized water before measurement. The particle sizes of SMEDDS and Sandimmun Neoral ${ }^{\circledR}$ were determined after microemulsifying in deionized water. The zeta potential was also determined by Zetasizer $\mathrm{Nano}^{\circledR}$ at $25^{\circ} \mathrm{C}$, and samples were placed into folded capillary cells integrated with gold electrodes. Three replicated samples were measured and each sample was conducted in triple measurements.

\section{Transmission electron microscopy}

The morphology of PLGA NPs, NLCs, SMEDDS, and Sandimmun Neoral ${ }^{\circledR}$ was observed by transmission electron microscopy (TEM). The microemulsion droplets were obtained by microemulsifying SMEDDS and Sandimmun Neoral ${ }^{\circledR}$ in deionized water. Then the PLGA NPs, NLCs suspension, and microemulsion droplets were placed on copper grids and negatively stained with $2 \%(\mathrm{w} / \mathrm{v})$ phosphotungstic acid for 5 minutes at room temperature. Finally, the grids bearing PLGA NPs, NLCs, and microemulsion droplets were observed with a JEM-1230 TEM (JEOL, Tokyo, Japan).

\section{Determination of entrapment efficiency of PLGA NPs and NLCs}

PLGA NPs were centrifuged at $30,000 \times g$ for 30 minutes to separate NPs with free drugs. The precipitant was dissolved in acetonitrile, which was analyzed by HPLC to determine the amount of CyA incorporated into NPs. ${ }^{30}$ The entrapment efficiency (EE) of PLGA NPs was calculated by the following equation.

$$
\mathrm{EE}(\%)=\frac{\text { Amount of CyA incorporated into NPs }}{\text { Total amount of CyA }} \times 100 \%
$$

The EE of NLCs was determined by ultrafiltration method and utilized centrifugal filter tubes with a molecular weight cutoff of $100 \mathrm{kDa} .{ }^{31}$ The concentration of total CyA in NLCs suspension and ultrafiltrate (free CyA) was analyzed by HPLC. The EE was calculated using the following equation.

$$
\mathrm{EE}(\%)=\frac{\text { Total amount of CyA }- \text { Amount of free } \mathrm{CyA}}{\text { Total amount of } \mathrm{CyA}} \times 100 \%
$$

\section{Release test}

The release test of NLCs, PLGA NPs, SMEDDS, and commercial Sandimmun Neoral ${ }^{\circledR}$ capsules were evaluated by a dynamic dialysis method in a ZRS-8G dissolution tester (Tianjin, People's Republic of China). ${ }^{27}$ NLCs, PLGA NPs, and SMEDDS dispersions containing $6 \mathrm{mg}$ CyA were sealed in dialysis bags (molecular weight cutoff 14,000; Millipore, Boston, MA, USA), while Sandimmun Neoral $^{\circledR}$ capsules were dispersed in $12.5 \mathrm{~mL}$ deionized water, $3 \mathrm{~mL}$ of which was sealed in dialysis bags. In order to maintain the sink condition, phosphate-buffered saline (PBS, pH 6.8) with $0.2 \%(\mathrm{w} / \mathrm{v})$ sodium lauryl sulfate was used as a release medium. The dialysis bags were immersed in $100 \mathrm{~mL}$ release medium and stirred at a speed of $100 \mathrm{rpm}$. The $1 \mathrm{~mL}$ of medium was withdrawn at time intervals of 0.25 hour, 0.50 hour, 1.0 hour, 2.0 hours, 3.0 hours, 6.0 hours, and 12.0 hours, and then determined by HPLC.

\section{Oral bioavailability study}

Six beagle dogs (adult male, $15.0 \pm 0.5 \mathrm{~kg}$ ) used in the experiments received care in compliance with the Principles of Laboratory Animal Care and the Guide for the Care and 
Use of Laboratory Animals. Prior to experiment, the dogs were fasted overnight and allowed free access to water. The four carriers (NLCs, PLGA NPs, SMEDDS, and Sandimmun Neoral ${ }^{\circledR}$ ) were respectively administered to the dogs by oral gavage at an equivalent dose of $10 \mathrm{mg} / \mathrm{kg} \mathrm{CyA}$ and the washout period was set as 1 week. After administration, about $3 \mathrm{~mL}$ blood was collected through the hind leg vein into heparinized tubes at 0.25 hour, 0.5 hour, 1.0 hour, 1.5 hours, 2.0 hours, 2.5 hours, 3.0 hours, 4.0 hours, 6.0 hours, 8.0 hours, 12.0 hours, 24.0 hours, 36.0 hours, and 48.0 hours. Blood samples were stored at $-20^{\circ} \mathrm{C}$ until analysis. CyA in whole blood was extracted by liquid-liquid extraction procedures established in our previous study, ${ }^{30}$ and concentration of CyA was determined by HPLC.

DAS professional software version 2.0 (Anhui, People's Republic of China) was employed to calculate pharmacokinetic parameters. The pharmacokinetic parameters, such as peak plasma concentration $\left(\mathrm{C}_{\max }\right)$, the time to maximum plasma concentration $\left(\mathrm{T}_{\max }\right)$, and the area under the concentration-time curve (AUC) between 0 hour and 48 hours $\left(\mathrm{AUC}_{0-48}\right)$ were obtained by noncompartmental analysis based on statistical moment theory.

\section{HPLC determination of CyA}

Both in vitro and in vivo samples were determined using an HPLC system (LC-10 ATvp, Shimadzu, Japan) comprising a binary pump, a tunable ultraviolet detector, and a column heater. The analytical column was a C18 column (Venusil XBP, $5 \mu \mathrm{m}, 4.6 \times 150 \mathrm{~mm}$; Agela, Tianjin, People's Republic of China) guarded with a refillable precolumn $(\mathrm{C} 18,2.0 \times 20 \mathrm{~mm}$; Alltech, Lexington, KY, USA). The ultraviolet detector was set at a wavelength of $287 \mathrm{~nm}$. The column temperature was set to $70^{\circ} \mathrm{C}$. For in vitro determination of CyA, the mobile phase consists of acetonitrile/water/tert-butyl methyl ether/ phosphoric acid $(60 / 35 / 5 / 0.1, \mathrm{v} / \mathrm{v} / \mathrm{v} / \mathrm{v})$ at a flow rate of $1.0 \mathrm{~mL} / \mathrm{minute}$. However, the mobile phase was a mixture of acetonitrile/water/tert-butyl methyl ether/phosphoric acid $(525 / 425 / 50 / 1, \mathrm{v} / \mathrm{v} / \mathrm{v} / \mathrm{v})$ at a flow rate of $1.5 \mathrm{~mL} / \mathrm{minute}$ for in vivo determination. ${ }^{16}$

\section{Statistical analysis}

All data were expressed as mean \pm standard deviation. One-way analysis of variance followed by Tukey's test was performed to assess the statistical significance of difference. Results with $P<0.05$ were considered statistically significant.

\section{Results}

\section{Preparation and characterization of PLGA NPs, NLCs, and SMEDDS}

CyA-loaded PLGA NPs, NLCs, and SMEDDS were prepared successfully. The particle size, polydispersion index (PDI), and zeta potential of each vehicle are summarized in Table 1.

The order of particle size of obtained nanovehicles was PLGA NPs $>$ NLCs $>$ SMEDDS. PLGA NPs are rigid NPs, and thus it is difficult to decrease particle size to $<100 \mathrm{~nm}$ by high-pressure homogenization. However, NLCs are NPs that are between "soft" and "hard", as they are composed of liquid and solid lipids. The formulation components are different between self-made SMEDDS and Sandimmun Neoral ${ }^{\circledR}$. Sandimmun Neoral ${ }^{\circledR}$ is composed of alcohol, corn oil (mono-di-triglycerides), Cremophor RH40 (polyoxyl 40 hydrogenated castor oil), and DL- $\alpha$-tocopherol. The formulation components of self-made SMEDDS include Labrafil M 1944 CS (oleoyl macrogoglycerides), Cremophor EL (polyoxyl 35 castor oil), and Trancutol P (diethylene glycol monoethyl ether), which removes the ethanol in formulation. Nevertheless, the particle size of self-made SMEDDS was not significantly different from that of Sandimmun Neoral ${ }^{\circledR}$ after dispersing in deionized water. The PDI of all vehicles was lower than 0.24 , which indicated relatively narrow particle-size distribution. Owing to the fatty acid in lipids of NLCs, NLCs had a higher negative charge than other vehicles. High zeta potential is necessary to keep the in vitro stability of nanosuspensions. ${ }^{32}$ The other vehicles had negative charge of lower than $-5 \mathrm{mV}$, but the particles size and PDI of all vehicles had no significant increase within 24 hours (Figure 1). The EEs of PLGA NPs and NLCs were

Table I Formulation characterization of CyA-loaded PLGA NPs, NLCs, SMEDDS, and commercial Sandimmun Neoral ${ }^{\circledR}$ capsule $(n=3)$

\begin{tabular}{lllll}
\hline Vehicle & Size $(\mathbf{n m})$ & PDI & Zeta potential $(\mathbf{m V})$ & EE $(\%)$ \\
\hline PLGA NPs & $182.2 \pm 12.8$ & $0.15 \pm 0.04$ & $-5.0 \pm 3.4$ & $87.6 \pm 1.6$ \\
NLCs & $89.7 \pm 9.0$ & $0.24 \pm 0.02$ & $-16.2 \pm 3.6$ & $80.3 \pm 0.6$ \\
SMEDDS & $26.9 \pm 1.9$ & $0.12 \pm 0.05$ & $-3.1 \pm 1.1$ & - \\
Sandimmun Neoral ${ }^{\circledR}$ & $30.0 \pm 0.1$ & $0.14 \pm 0.02$ & $-4.2 \pm 1.1$ & - \\
\hline
\end{tabular}

Abbreviations: CyA, cyclosporine A; EE, entrapment efficiency; NP, nanoparticle; NLC, nanostructured lipid carrier; PDI, polydispersion index; PLGA, poly(lactic-coglycolic acid); SMEDDS, self-microemulsifying drug-delivery systems. 
A

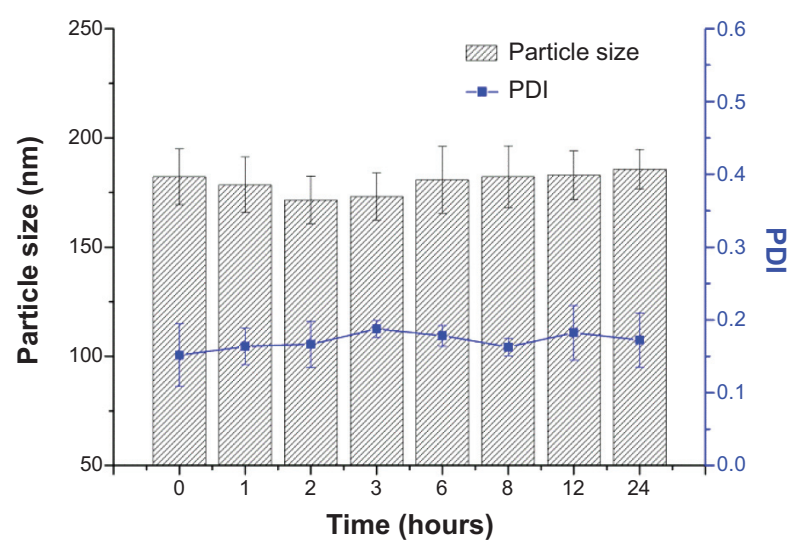

B

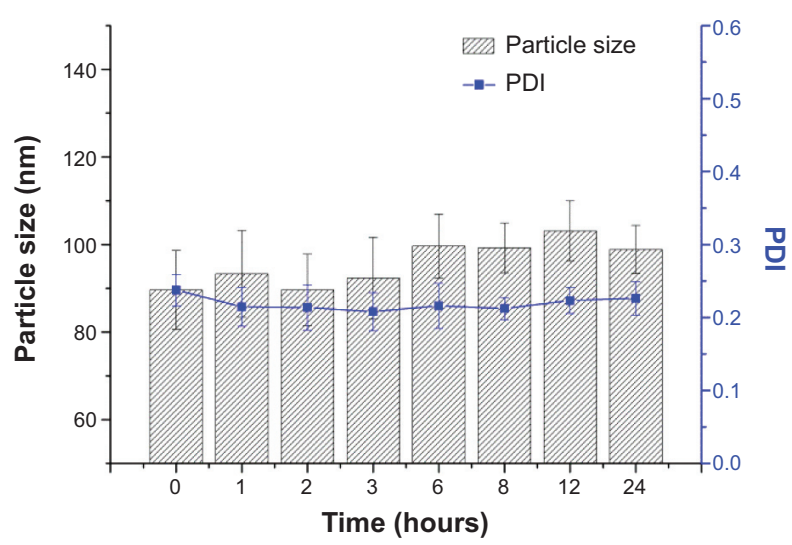

C

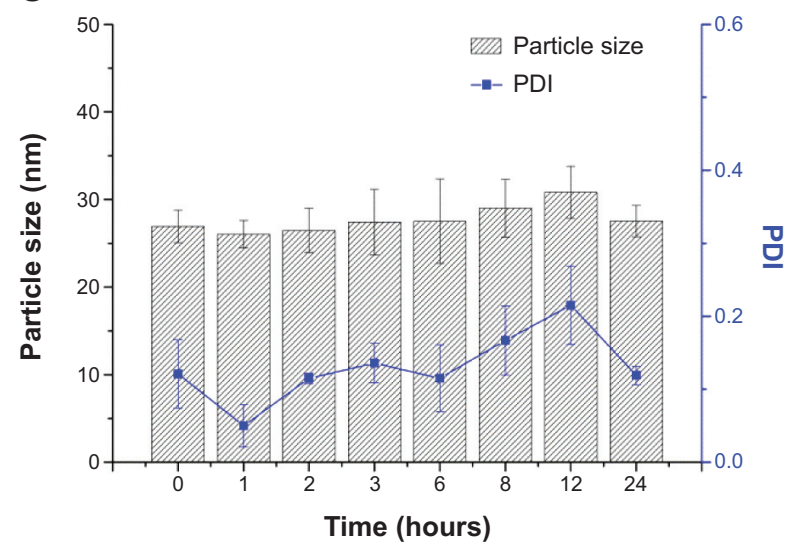

Figure I Change in particle size and PDI within 24 hours $(n=3)$.

Notes: (A) PLGA NPs, (B) NLCs, and (C) SMEDDS.

Abbreviations: NP, nanoparticle; NLC, nanostructured lipid carrier; PDI, polydispersion index; PLGA, poly(lactic-co-glycolic acid); SMEDDS, self-microemulsifying drugdelivery systems.

$87.6 \% \pm 1.6 \%$ and $80.3 \% \pm 0.6 \%$, respectively, suggesting efficient encapsulation of CyA.

\section{Morphology}

TEM was employed to observe the morphology of PLGA NPs, NLCs, and microemulsions reconstituted from SMEDDS and Sandimmun Neoral ${ }^{\circledR}$ (Figure 2). Both the PLGA NPs (Figure 2A) and the NLCs (Figure 2B) were spherical in shape and similar in size, according to the results of dynamic light scattering. The particle size of PLGA NPs was more well-distributed than that of NLCs. Figure 2C and $\mathrm{D}$ demonstrate the morphology of microemulsions formed by self-made SMEDDS and Sandimmun Neoral ${ }^{\circledR}$, respectively, and indicate that there are no significant differences between them.

\section{Release test}

Release profiles of the four vehicles were shown in Figure 3. CyA almost cannot release from the four vehicles. In this study, the sink condition was achieved by the addition of $0.2 \%$ sodium lauryl sulfate in PBS. In addition, CyA in solution could diffuse completely across the dialysis bag within 1 hour, suggesting a negligible hindering effect of the dialysis bag on release of CyA. ${ }^{27,33} \mathrm{CyA}$ cannot release from NLCs or SMEDDS, as CyA has good affinity for lipids. It is generally thought that NLCs and SMEDDS release drug molecules by digestion with lipase when they enter into the GIT..$^{34,35}$ However, PLGA NPs release drugs by erosion, which may take a few days or months. ${ }^{36,37}$ Therefore, cumulative release amount of drug from carriers in PBS was extremely low within 12 hours.

\section{Oral bioavailability}

To investigate the role of different vehicles in enhancing oral absorption of CyA, oral bioavailability of CyA-loaded PLGA NPs, NLCs, and SMEDDS in beagle dogs were compared. The mean whole-blood CyA concentrations versus time plots for the four vehicles are shown in Figure 4, and 

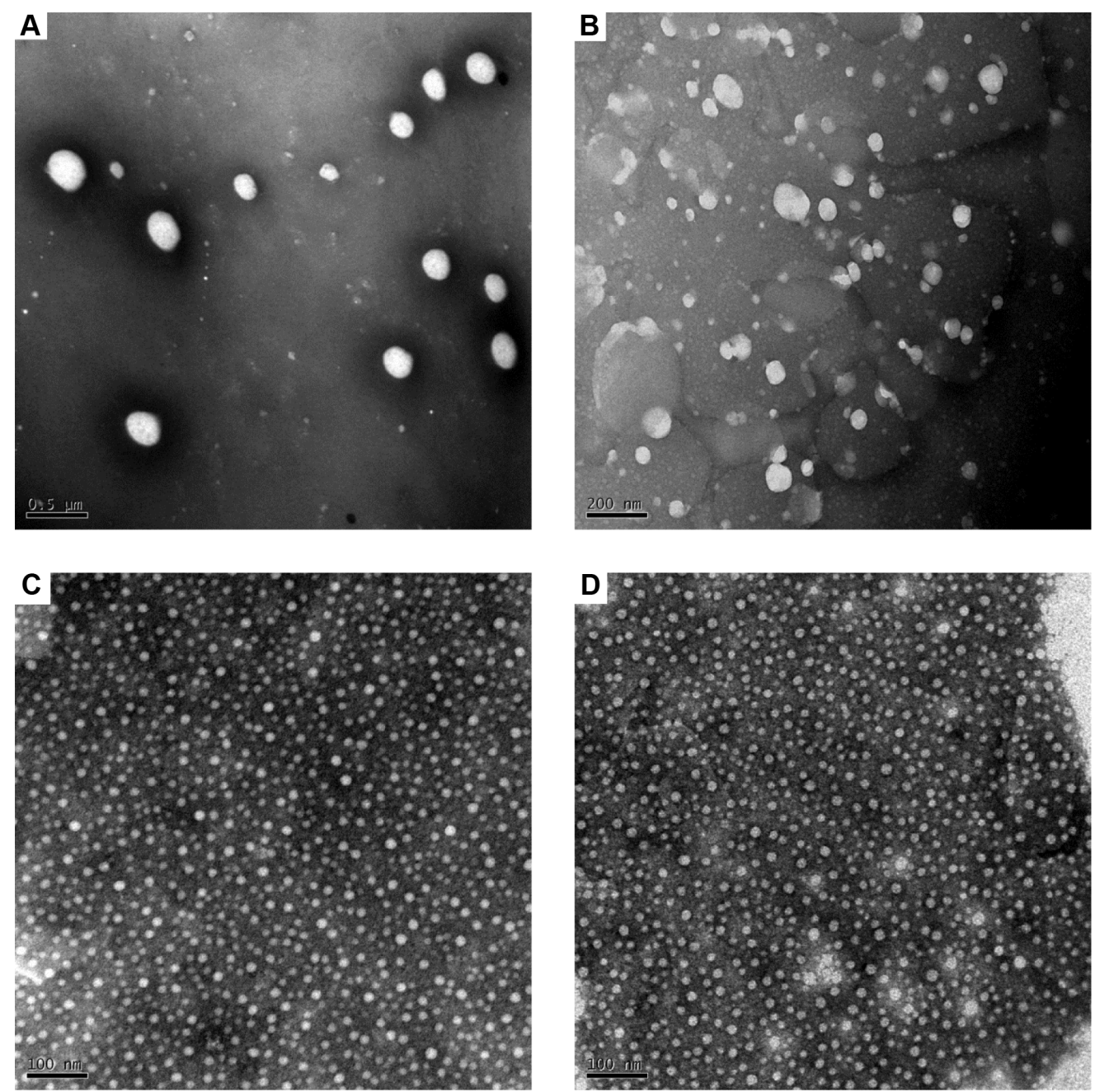

Figure 2 Morphology of CyA-loaded delivery systems observed by transmission electron microscopy.

Notes: (A) PLGA NPs, (B) NLCs, (C) microemulsions droplets formed by SMEDDS, and (D) Sandimmun Neoral ${ }^{\otimes}$.

Abbreviations: CyA, cyclosporine A; NP, nanoparticle; NLC, nanostructured lipid carrier; PLGA, poly(lactic-co-glycolic acid); SMEDDS, self-microemulsifying drug-delivery systems.

the pharmacokinetic parameters obtained by the statistical moment method are shown in Table 2.

After oral administration, the $\mathrm{T}_{\text {max }}$ of CyA in Sandimmun Neoral ${ }^{\circledR}$ was $1.92 \pm 0.58$ hours, which was similar to previous reports. ${ }^{33,38}$ Although the $\mathrm{T}_{\max }$ of NLCs, SMEDDS, and PLGA NPs was lower than that of the reference, there was no significant difference in $\mathrm{T}_{\text {max }}$ after oral administration of the four vehicles, mainly due to the larger individual variation. In terms of $\mathrm{C}_{\max }$, NLCs showed the maximum value among the four vehicles $(2.32 \pm 1.05 \mu \mathrm{g} / \mathrm{mL})$ but was not significantly different from Sandimmun Neoral ${ }^{\circledR}(1.76 \pm 0.77 \mu \mathrm{g} / \mathrm{mL})$. In contrast, the $\mathrm{C}_{\max }$ of PLGA NPs was lower than that of others and statistically different from Sandimmun Neoral ${ }^{\circledR}$ $(P<0.05)$. Similarly, NLCs obtained statistically higher $\mathrm{AUC}_{0-48}$ than PLGA NPs, but no statistical difference with Sandimmun Neoral ${ }^{\circledR}$ or SMEDDS.

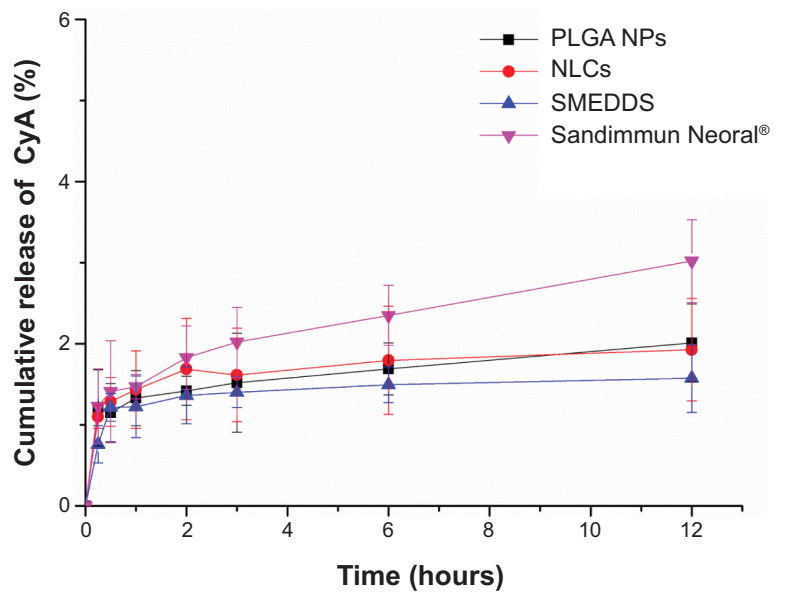

Figure 3 In vitro release profiles of PLGA NPs, NLCs, SMEDDS, and commercial Sandimmun Neora ${ }^{\circledR}$ capsule $(n=3)$.

Abbreviations: CyA, cyclosporine A; NP, nanoparticle; NLC, nanostructured lipid carrier; PLGA, poly(lactic-co-glycolic acid); SMEDDS, self-microemulsifying drugdelivery systems. 


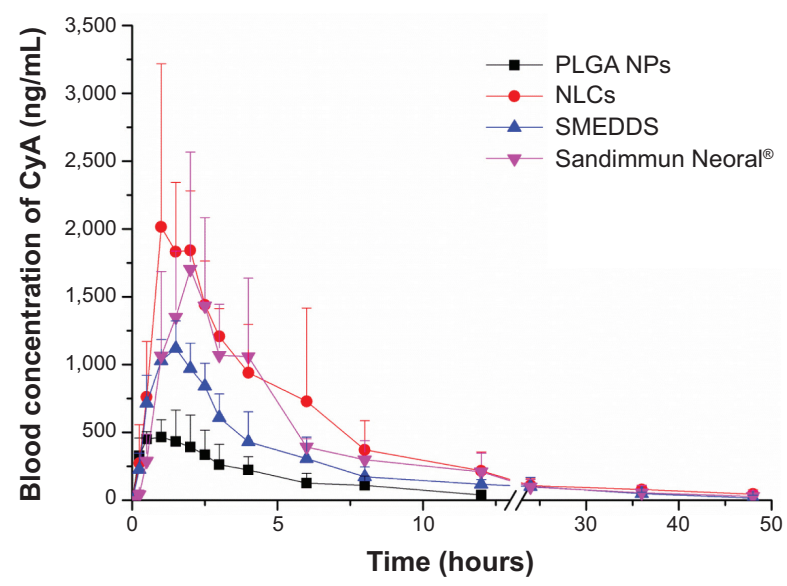

Figure 4 Mean blood concentration-time plot of CyA in beagle dogs after oral administration of CyA-loaded PLGA NPs, NLCs, SMEDDS, and Sandimmun Neoral ${ }^{\circledast}$ capsules $(\mathrm{n}=6)$.

Abbreviations: CyA, cyclosporine A; NP, nanoparticle; NLC, nanostructured lipid carrier; PLGA, poly(lactic-co-glycolic acid); SMEDDS, self-microemulsifying drugdelivery systems.

\section{Discussion}

The improvement in BCS IV drug bioavailability was dependent not only on increase of dissolution in GIT but also on enhancement of permeability across intestinal epithelia. PLGA NPs are polymeric NPs, which were not degraded in GIT within a short time and could not release drug rapidly. However, PLGA NPs can adhere to mucus to increase the retention time of drugs in GIT due to their very small particle size and inhibit the efflux of P-glycoprotein to enhance the transport of CyA across the intestinal epithelia. ${ }^{39}$ Furthermore, intact PLGA NPs may be endocytosed by intestinal epithelia or $\mathrm{M}$ cells $\mathrm{s}^{40,41}$ and can protect the drug from degradation in GIT. ${ }^{42}$ Nevertheless, it is well known that adsorptive endocytosis is initiated by electrostatic forces, by which positively charged particulate vehicles can readily adhere to the negatively charged cell surface of intestinal mucosa. ${ }^{43}$ In our study, PLGA NPs were prepared without any chemical modification and displayed negative charge. Meanwhile, it is generally believed that endocytosis of intestinal epithelial is a slow and infrequent process compared with passive transport. ${ }^{44,45}$ Consequently, PLGA NPs showed no significant enhanced ability of oral absorption, indicating that uptake of NPs by endocytosis of intestinal epithelia cannot increase oral bioavailability of BCS IV drugs to a large extent. Although the endocytosis may be also affected by particle size, one study suggested the uptake of PLGA NPs by intestinal epithelia was not significantly different for particles sized $100-200 \mathrm{~nm} .{ }^{46}$ However, our previous study concluded that a single mechanism of increased dissolution, such as solid dispersion or nanocrystals, cannot sufficiently promote the bioavailability of a BCS IV drug. ${ }^{47}$

With respect to lipid-based nanocarriers, not only did they achieved a higher $\mathrm{C}_{\max }$ value in an earlier absorption stage, but they also maintained a higher level of drug concentration over the whole absorption process, compared with PLGA NPs. Hence, we concluded that lipid-based nanocarriers achieved a better bioavailability-enhancing effect, which may be mainly due to a combined promotion mechanisms as follows.

Firstly, the ability to adhere to gut wall should be taken into account. Though negatively charged particulates and droplets should encounter electrostatic repulsion by the negatively charged mucus gel layer of enterocytes, which is unfavorable to the absorption of CyA, ultrafine dispersions within nanometer range were realized in all three lipid nanocarriers and this endows them with a tremendous specific surface area and thus the possession of adhesion to gut wall, ${ }^{23}$ which is helpful to oral absorption.

Secondly, lipid-based nanocarriers, such as NLCs or SMEDDS, would be degraded by lipase to transform into a series of secondary derivatives (eg, mixed micelles, cubic and hexagonal NPs, vesicular carriers) with the help of endogenous bile salts and phospholipids in GIT. Drugs are able to be solubilized in these secondary derivatives, ${ }^{16,27,33}$ which is very helpful to drug absorption. Meanwhile, reports have confirmed that the cellular uptake was size-dependent and the endocytosis was mediated via clathrin as particle diameter was smaller than $200 \mathrm{~nm}$, which may be helpful to increase oral bioavailability. ${ }^{44,48}$

Table 2 Pharmacokinetic parameters after oral administration of CyA-loaded PLGA NPs, NLCs, SMEDDS, and commercial Sandimmun Neoral ${ }^{\circledR}$ capsule $(n=6)$

\begin{tabular}{lllll}
\hline Vehicles & $\mathbf{T}_{\max }$ (hours) & $\mathbf{C}_{\max }(\mu \mathrm{g} / \mathrm{mL})$ & AUC $_{0-48}(\mu \mathrm{g} *$ hours $/ \mathrm{mL})$ & RBA (\%) \\
\hline PLGA NPs & $1.05 \pm 0.94$ & $0.56 \pm 0.15^{*}$ & $2.21 \pm 0.96 *$ & $22.71 \pm 6.73$ \\
NLCs & $1.40 \pm 0.65$ & $2.32 \pm 1.05$ & $11.43 \pm 3.27$ & $111.83 \pm 40.0$ \\
SMEDDS & $1.50 \pm 0.35$ & $1.12 \pm 0.20$ & $6.96 \pm 1.63$ & $73.57 \pm 25.65$ \\
Sandimmun Neoral ${ }^{\circledR}$ & $1.92 \pm 0.58$ & $1.76 \pm 0.77$ & $10.28 \pm 4.25$ & - \\
\hline
\end{tabular}

Note: $* P<0.05$ compared with Sandimmun Neora ${ }^{\circledR}$ capsule.

Abbreviations: AUC, area under the concentration-time curve between 0 and 48 hours; CyA, cyclosporine A; NP, nanoparticle; NLC, nanostructured lipid carriers; PLGA, poly(lactic-co-glycolic acid); RBA, relative bioavailability calculated with Sandimmun Neoral as reference; SMEDDS, self-microemulsifying drug-delivery systems; $T_{\text {max }}$, time to maximum plasma concentration; $\mathrm{C}_{\max }$, peak plasma concentration. 
Thirdly, intestinal lymphatic drug transport may be recruited by lipid-based vehicles and prolong the later absorption period of CyA (about 6-8 hours after administration in our study), since lymphatic transport is slow, sporadic, and able to avoid first-pass metabolism. ${ }^{49}$

It should be noted that lipid excipient like Cremophor ${ }^{\circledR}$ EL added in SMEDDS should be digested as well. Digestion of surfactants may cause loss of capacity to keep the drug compound in solution, especially when that excipient is included in relatively high proportion $(46.67 \%$ of total excipients in our study)..$^{50,51}$

\section{Conclusion}

Through the development of a variety of drug nanocarriers (including PLGA NPs, NLCs, and SMEDDS), the oral bioavailability of CyA was well studied. Compared with commercial Sandimmun Neoral ${ }^{\circledR}$, lipid-based nanocarriers (NLCs and SMEDDS) achieved similar oral bioavailability, but polymeric NPs (PLGA NPs) failed to improve the oral bioavailability of CyA to a great extent. It was suggested that lipid-based drug-delivery systems were more advantageous than others in improving oral absorption of BCS IV drugs, due to the multiple absorption enhancement mechanisms.

\section{Acknowledgments}

We are grateful for the financial support from Shanghai Commission of Science and Technology (14JC1490300, $11 \mathrm{~nm} 0506700)$. Dr Wu would also want to thank Shanghai Commission of Education (10SG05) and Ministry of Education (NCET-11-0114) for personnel fostering support.

\section{Disclosure}

The authors report no conflicts of interest in this work.

\section{References}

1. Merisko-Liversidge EM, Liversidge GG. Drug nanoparticles: formulating poorly water-soluble compounds. Toxicol Pathol. 2008;36(1): 43-48.

2. Kawabata Y, Wada K, Nakatani M, Yamada S, Onoue S. Formulation design for poorly water-soluble drugs based on biopharmaceutics classification system: basic approaches and practical applications. Int $J$ Pharm. 2011;420(1):1-10.

3. Amidon GL, Lennernäs H, Shah VP, Crison JR. A theoretical basis for a biopharmaceutic drug classification: the correlation of in vitro drug product dissolution and in vivo bioavailability. Pharm Res. 1995;12(3): 413-420.

4. Iqbal MA, Md S, Sahni JK, Baboota S, Dang S, Ali J. Nanostructured lipid carriers system: recent advances in drug delivery. J Drug Target. 2012;20(10):813-830.

5. Cao X, Gibbs ST, Fang L, et al. Why is it challenging to predict intestinal drug absorption and oral bioavailability in human using rat model. Pharm Res. 2006;23(8):1675-1686.
6. Xu Y, Liu X, Lian R, et al. Enhanced dissolution and oral bioavailability of aripiprazole nanosuspensions prepared by nanoprecipitation/homogenization based on acid-base neutralization. Int J Pharm. 2012;438(1-2): 287-295.

7. Fatma S, Talegaonkar S, Iqbal Z, et al. Novel flavonoid-based biodegradable nanoparticles for effective oral delivery of etoposide by P-glycoprotein modulation: an in vitro, ex vivo and in vivo investigations. Drug Deliv. 2014:1-12.

8. Liu YO, Fan JM, Wang XQ, Zhang Q. Preparation of sorafenib selfmicroemulsifying drug delivery system and its relative bioavailability in rats. J Chin Pharm Sci. 2011;20(2):164-170.

9. Wang S, Ye T, Zhang X, Yang R, Yi X. Myricetin loaded in microemulsion for oral drug delivery: formulation optimization, intestinal absorption in situ recirculation and in-vivo evaluation. Asian J Pharm Sci. 2012;7(5):293-300.

10. Hariharan S, Bhardwaj V, Bala I, Sitterberg J, Bakowsky U, Ravi Kumar MN. Design of estradiol loaded PLGA nanoparticulate formulations: a potential oral delivery system for hormone therapy. Pharm Res. 2006;23(1):184-195.

11. Arbós P, Campanero MA, Arangoa MA, Renedo MJ, Irache JM. Influence of the surface characteristics of PVM/MA nanoparticles on their bioadhesive properties. J Control Release. 2003;89(1):19-30.

12. Joshi G, Kumar A, Sawant K. Enhanced bioavailability and intestinal uptake of Gemcitabine $\mathrm{HCl}$ loaded PLGA nanoparticles after oral delivery. Eur J Pharm Sci. 2014;60:80-89.

13. Griffin BT, O’Driscoll CM. Opportunities and challenges for oral delivery of hydrophobic versus hydrophilic peptide and proteinlike drugs using lipid-based technologies. Ther Deliv. 2011;2(12): 1633-1653.

14. Pouton CW. Lipid formulations for oral administration of drugs: nonemulsifying, self-emulsifying and 'self-microemulsifying' drug delivery systems. Eur J Pharm Sci. 2000;11 Suppl 2:S93-S98.

15. Nanjwade BK, Patel DJ, Udhani RA, Manvi FV. Functions of lipids for enhancement of oral bioavailability of poorly water-soluble drugs. Sci Pharm. 2011;79(4):705-727.

16. Lei Y, Qi J, Nie S, et al. Solid self-nanoemulsifying cyclosporine A pellets prepared by fluid-bed coating: stability and bioavailability study. J Biomed Nanotechnol. 2012;8(3):515-521.

17. Italia JL, Bhatt DK, Bhardwaj V, Tikoo K, Kumar MN. PLGA nanoparticles for oral delivery of cyclosporine: nephrotoxicity and pharmacokinetic studies in comparison to Sandimmune Neoral. J Control Release. 2007;119(2):197-206.

18. Beauchesne PR, Chung NS, Wasan KM. Cyclosporine A: a review of current oral and intravenous delivery systems. Drug Dev Ind Pharm. 2007;33(3):211-220.

19. Onoue S, Sato H, Ogawa K, et al. Improved dissolution and pharmacokinetic behavior of cyclosporine A using high-energy amorphous solid dispersion approach. Int J Pharm. 2010;399(1-2):94-101.

20. Wacher VJ, Wu CY, Benet LZ. Overlapping substrate specificities and tissue distribution of cytochrome P450 3A and P-glycoprotein: implications for drug delivery and activity in cancer chemotherapy. Mol Carcinog. 1995;13(3):129-134.

21. Sharma P, Varma MV, Chawla HP, Panchagnula R. Relationship between lipophilicity of BCS class III and IV drugs and the functional activity of peroral absorption enhancers. Farmaco. 2005;60(11-12): 870-873.

22. Tjia JF, Webber IR, Back DJ. Cyclosporin metabolism by the gastrointestinal mucosa. Br J Clin Pharmacol. 1991;31(3):344-346.

23. Tarr BD, Yalkowsky SH. Enhanced intestinal absorption of cyclosporine in rats through the reduction of emulsion droplet size. Pharm Res. 1989;6(1):40-43.

24. Sato H, Kawabata Y, Yuminoki K, et al. Comparative studies on physicochemical stability of cyclosporine A-loaded amorphous solid dispersions. Int J Pharm. 2012;426(1-2):302-306.

25. Liu C, Zhu S, Zhou Y, Wei Y, Pei Y. In situ intestinal absorption of cyclosporine A solid dispersion in rats. Drug Dev Ind Pharm. 2008; 34(6):627-631. 
26. Nakarani M, Patel P, Patel J, Patel P, Murthy RS, Vaghani SS Cyclosporine a-nanosuspension: formulation, characterization and in vivo comparison with a marketed formulation. Sci Pharm. 2010; 78(2):345-361.

27. Guan P, Lu Y, Qi J, et al. Enhanced oral bioavailability of cyclosporine A by liposomes containing a bile salt. Int J Nanomedicine. 2011;6: 965-974.

28. Müller RH, Runge S, Ravelli V, Mehnert W, Thünemann AF, Souto EB. Oral bioavailability of cyclosporine: solid lipid nanoparticles (SLN) versus drug nanocrystals. Int J Pharm. 2006;317(1):82-89.

29. Tian Z, Yi Y, Yuan H, et al. Solidification of nanostructured lipid carriers (NLCs) onto pellets by fluid-bed coating: preparation, in vitro characterization and bioavailability in dogs. Powder Technol. 2013;247(Complete):120-127.

30. Lei Y, Lu Y, Qi J, et al. Solid self-nanoemulsifying cyclosporin A pellets prepared by fluid-bed coating: preparation, characterization and in vitro redispersibility. Int J Nanomedicine. 2011;6:795-805.

31. Anand P, Nair HB, Sung B, et al. Design of curcumin-loaded PLGA nanoparticles formulation with enhanced cellular uptake, and increased bioactivity in vitro and superior bioavailability in vivo. Biochem Pharmacol. 2010;79(3):330-338.

32. Mora-Huertas CE, Fessi H, Elaissari A. Polymer-based nanocapsules for drug delivery. Int J Pharm. 2010;385(1-2):113-142.

33. Lai J, Lu Y, Yin Z, Hu F, Wu W. Pharmacokinetics and enhanced oral bioavailability in beagle dogs of cyclosporine A encapsulated in glyceryl monooleate/poloxamer 407 cubic nanoparticles. Int J Nanomedicine. 2010;5:13-23.

34. Goddeeris C, Coacci J, Van den Mooter G. Correlation between digestion of the lipid phase of smedds and release of the anti-HIV drug UC 781 and the anti-mycotic drug enilconazole from smedds. Eur J Pharm Biopharm. 2007;66(2):173-181.

35. Williams HD, Trevaskis NL, Yeap YY, Anby MU, Pouton CW, Porter CJ. Lipid-based formulations and drug supersaturation: harnessing the unique benefits of the lipid digestion/absorption pathway. Pharm Res. 2013;30(12):2976-2992.

36. Sahana DK, Mittal G, Bhardwaj V, Kumar MN. PLGA nanoparticles for oral delivery of hydrophobic drugs: influence of organic solvent on nanoparticle formation and release behavior in vitro and in vivo using estradiol as a model drug. J Pharm Sci. 2008;97(4): $1530-1542$.

37. Zhao B, Yang LJ, Wang JC, Zhang Q. Studies on in vitro release of cyclosporine A-loaded microspheres. J Chin Pharm Sci. 2007;16(4): 252-256.

38. Liu C, Wu J, Shi B, Zhang Y, Gao T, Pei Y. Enhancing the bioavailability of cyclosporine a using solid dispersion containing polyoxyethylene (40) stearate. Drug Dev Ind Pharm. 2006;32(1):115-123.
39. Ling G, Zhang P, Zhang W, et al. Development of novel self-assembled DS-PLGA hybrid nanoparticles for improving oral bioavailability of vincristine sulfate by P-gp inhibition. J Control Release. 2010;148(2): 241-248.

40. Gupta PN, Khatri K, Goyal AK, Mishra N, Vyas SP. M-cell targeted biodegradable PLGA nanoparticles for oral immunization against hepatitis B. J Drug Target. 2007;15(10):701-713.

41. Sarti F, Perera G, Hintzen F, et al. In vivo evidence of oral vaccination with PLGA nanoparticles containing the immunostimulant monophosphoryl lipid A. Biomaterials. 2011;32(16):4052-4057.

42. Beloqui A, Solinís MÁ, Gascón AR, del Pozo-Rodríguez A, des Rieux A, Préat V. Mechanism of transport of saquinavir-loaded nanostructured lipid carriers across the intestinal barrier. J Control Release. 2013;166(2):115-123.

43. Martin-Banderas L, Durán-Lobato M, Muñoz-Rubio I, Alvarez-Fuentes J, Fernández-Arevalo M, Holgado MA. Functional PLGA NPs for oral drug delivery: recent strategies and developments. Mini Rev Med Chem. 2013;13(1):58-69.

44. Wang T, Bai J, Jiang X, Nienhaus GU. Cellular Uptake of nanoparticles by membrane penetration: a study combining confocal microscopy with FTIR spectroelectrochemistry. ACS Nano. 2012;6(2):1251-1259.

45. Vácha R, Martinez-Veracoechea FJ, Frenkel D. Receptor-mediated endocytosis of nanoparticles of various shapes. Nano Lett. 2011; 11(12):5391-5395.

46. Xu A, Yao M, Xu G, et al. A physical model for the size-dependent cellular uptake of nanoparticles modified with cationic surfactants. Int J Nanomedicine. 2012;7:3547-3554.

47. Wang K, Lu Y, Qi J, Yin Z, W. W. Comparative study on oral bioavailability of cyclosporine A-loaded solid dispersion and nanosuspension. J Chin Pharm Sci. 2014;45(4):354-358.

48. Roger E, Lagarce F, Garcion E, Benoit JP. Lipid nanocarriers improve paclitaxel transport throughout human intestinal epithelial cells by using vesicle-mediated transcytosis. J Control Release. 2009;140(2): 174-181.

49. Sachs-Barrable K, Lee SD, Wasan EK, Thornton SJ, Wasan KM. Enhancing drug absorption using lipids: a case study presenting the development and pharmacological evaluation of a novel lipid-based oral amphotericin B formulation for the treatment of systemic fungal infections. Adv Drug Deliv Rev. 2008;60(6):692-701

50. Cuiné JF, McEvoy CL, Charman WN, et al. Evaluation of the impact of surfactant digestion on the bioavailability of danazol after oral administration of lipidic self-emulsifying formulations to dogs. J Pharm Sci. 2008;97(2):995-1012.

51. Larsen AT, Ohlsson AG, Polentarutti B, et al. Oral bioavailability of cinnarizine in dogs: relation to SNEDDS droplet size, drug solubility and in vitro precipitation. Eur J Pharm Sci. 2013;48(1-2):339-350.
International Journal of Nanomedicine

\section{Publish your work in this journal}

The International Journal of Nanomedicine is an international, peerreviewed journal focusing on the application of nanotechnology in diagnostics, therapeutics, and drug delivery systems throughout the biomedical field. This journal is indexed on PubMed Central, MedLine, CAS, SciSearch $®$, Current Contents $\AA /$ Clinical Medicine,

\section{Dovepress}

Journal Citation Reports/Science Edition, EMBase, Scopus and the Elsevier Bibliographic databases. The manuscript management system is completely online and includes a very quick and fair peer-review system, which is all easy to use. Visit http://www.dovepress.com/ testimonials.php to read real quotes from published authors. 